Provided for non-commercial research and education use. Not for reproduction, distribution or commercial use.

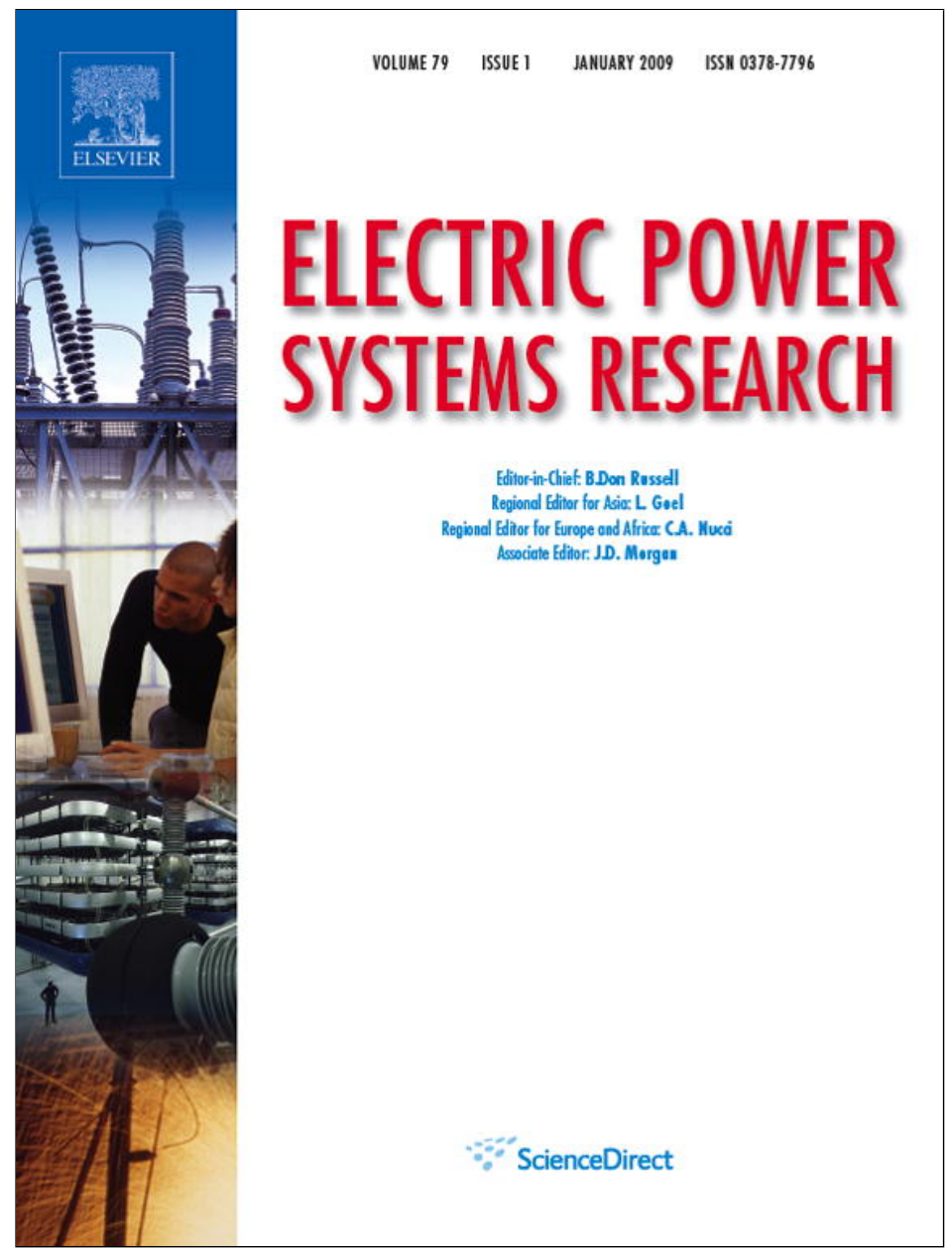

This article appeared in a journal published by Elsevier. The attached copy is furnished to the author for internal non-commercial research and education use, including for instruction at the authors institution and sharing with colleagues.

Other uses, including reproduction and distribution, or selling or licensing copies, or posting to personal, institutional or third party websites are prohibited.

In most cases authors are permitted to post their version of the article (e.g. in Word or Tex form) to their personal website or institutional repository. Authors requiring further information regarding Elsevier's archiving and manuscript policies are encouraged to visit:

http://www.elsevier.com/copyright 


\title{
An optimization approach for wind turbine commitment and dispatch in a wind park
}

\author{
Carlos F. Moyano ${ }^{c, *}$, João A. Peças Lopes ${ }^{\mathrm{a}, \mathrm{b}, 1}$ \\ a Instituto de Engenharia de Sistemas e Computadores do Porto, Portugal \\ ${ }^{\mathrm{b}}$ Faculdade de Engenharia da Universidade do Porto, Campus da FEUP, Rua Dr. Roberto Frias, 378 4200-465 Porto, Portugal \\ ' School of Engineering Systems, Faculty of Built Environment and Engineering, Queensland University of Technology, GPO Box 2434, Brisbane, QLD 4001, Australia
}

\section{A R T I C L E I N F O}

\section{Article history:}

Received 24 April 2007

Received in revised form 19 March 2008

Accepted 21 May 2008

Available online 9 July 2008

\section{Keywords:}

Wind power generation

Active and reactive power dispatch

Optimization strategies

\begin{abstract}
A B S T R A C T
This paper describes an operational optimization strategy to be adopted at the wind park control level, that enables defining the commitment of wind turbines and their active and reactive power outputs following requests from Wind Park Dispatch Centers, assuming that individual wind turbines short-term wind speed forecasts are known and are expressed as power availability. This operational strategy was also developed with a concern on the minimization of the connection/disconnection changes of the individual wind generators, for a given time horizon. When identifying the active/reactive dispatching policies, wind generators loading capabilities are also taken in account. This optimization tool is especially suited to manage large wind parks.
\end{abstract}

(C) 2008 Elsevier B.V. All rights reserved.

\section{Introduction}

The large increase of the penetration of wind generation in power systems is leading to the need of controlling wind parks output (active and reactive power), namely in situations where wind generation is delivering its energy to the market or when grid operational restrictions require a strict control over the output of these generation entities. Since a considerable share of this generation is geographically distributed in several wind parks, with different installed capacities, a hierarchical monitoring and control approach is being developed and adopted in several systems. This control approach involves, on top of the controlling pyramid, a Wind Park Dispatch Center controlling several wind parks that will be required to dispatch their wind energy conversion systems to fulfil the demands from the Central Dispatch Center.

As example, Wind Generation Dispatch Centers (acting as Generation Aggregation Agents) will be installed in the Portuguese system, adopting for this purpose a hierarchical control architecture. Having in mind that wind generation can inject power either in the transmission network or in the distribution grids, communication links must be established between the Wind Generation Dispatch Centers and the transmission system operator (TSO) or

\footnotetext{
* Corresponding author. Tel.: +61 73138 9125; fax: +61 731381516. E-mail addresses: carlos.moyano@qut.edu.au (C.F. Moyano), jpl@fe.up.pt (J.A. Peças Lopes).

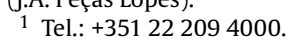

the distribution system operators (DSO) [1]. If one assumes also, the participation of wind generation in the market, similar communication links must be established with the market operator. Spain is presently already promoting the development of a similar architecture for wind generation dispatch control centres [2].

Under this scenario, wind generators are expected to be able to control their active and reactive power outputs. Double fed induction generators (DFIG) controlled by static converters and variable speed synchronous generators with full electronic grid interface are presently already capable of assuring such individual control capabilities [3].

It is also known that wind generators where reactive power generation can be controlled allow wind parks capacity to be further increased [4], since the transfer capacity of the wind park interconnection lines can be better used.

Regarding DFIG, a great variety of control strategies can be used in the operation of these machines as it can be seen from Refs. [3,5-9]. In these works, strategies to control active and reactive power wind park outputs also were proposed. In these researches wind park control models are built-up with a hierarchical modular structure: a central wind park controller sends out reference signals to each local wind turbine controller. Namely in Ref. [7] a dispatch module for a wind park is described, assuming however that wind generator's dispatch is performed as a proportional division of operator's request through all the wind turbines. Ref. [9] describes an optimization strategy to be adopted at the supervisory wind park control level with the objective of assuring that the wind park active and reactive power outputs fulfil the requests 
defined by system operators, assuming wind speeds constant and equal for all the wind turbines and without the possibility of connecting/disconnecting wind generators.

When dealing with the management strategy of a large wind park, wind power forecast are needed, in order to allow for a correct allocation of generation and to assign a confidence interval and risk indicators to the results obtained. Wind power forecasts can be obtained in a wind park for each wind turbine, adopting the methodology described in Ref. [19] that also takes into account the orography of the wind park site, provided that wind speeds from meteorological wind speed forecasts are available. A methodology for assessing on-line the prediction risk of short-term wind power forecasts, is described in Ref. [10]. This methodology consists in computing confidence intervals with a confidence level defined by the end-user and adapted for the wind power prediction problem taking into account the dependency of the errors on the level of predicted power.

The identification of an adequate operational strategy for the wind generators requires the consideration of the relation between active and reactive power in order to keep each generator operating inside their technical limits. On the other hand, if wind power generation is used to provide a voltage control and reactive power ancillary services, such situation must be taken into account when defining the wind park operational strategy. Ref. [11] describes the modelling of the conventional synchronous generators into reactive $\mathrm{OPF}$, and shows that the influence of a generator in the reactive power market changes with the load demand, influencing the voltage profile and also the maximum loadability limit of the system.

The present paper describes an optimization strategy, to be adopted at the wind park control level, to define the commitment of the wind turbines and their active and reactive set points following requests from the Wind Park Dispatch Centers, assuming that individual wind turbines short-term power generation forecasts are known and are expressed as active available power [19]. This approach was also developed with a concern on the minimization of the connection/disconnection of the machines for a given time horizon. The global optimization problem is formulated in two stages, in a similar way as in conventional generation systems, solving first the unit commitment problem, and them solving a nonlinear problem that allows obtaining active and reactive power set points for wind turbines. An example for a hypothetical wind park with 10 wind generators, presented in Fig. 1, is used in this paper to illustrate the presented approach.

\section{Optimization problem}

The problem of deciding which turbine will be committed, and identifying the active and reactive power output set points for each wind turbine in a wind park, following requests from a Wind Park Dispatch Center can be performed in a similar way as the one used in classical generation systems, through the solution of two interrelated optimization problems. A Unit Commitment problem is solved first, in order to determine the turbine schedule, and a technical dispatch is solved to find the set point values for each turbine at each period in a second step. In both of these problems, the demanded power is the power requested by the Wind Park Dispatch Center.

Wind parks production has some particularities that need to be considered. One of these special characteristics is that wind power has no generation cost, but is not always available. Therefore a short-term forecasting needs to be performed in order to know the availability of generation in the different wind turbines. In this work one has assumed that individual wind turbines short-term wind generation forecasts are known being expressed as power avail-

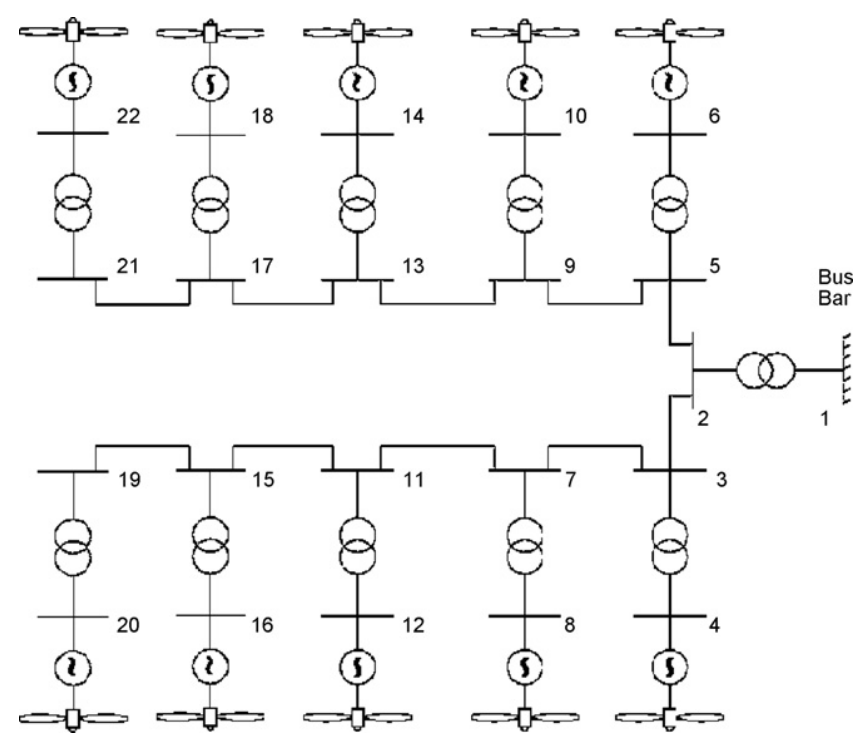

Fig. 1. Wind park schematic.

ability, as in Refs. [10,19], with an associated confidence interval. Another characteristic is that wind parks have a radial configuration that allows obtaining an estimate of the wind park internal active losses. The time horizon to be adopted can be considered according to the operational needs. However, since the forecast error increases as the time horizon increases, a short-term power forecast of less than $6 \mathrm{~h}$ is usually adopted.

\subsection{Unit commitment of wind turbines}

In order to identify the status (on-off) of each wind turbine $j$ at period $i$, binary variables $X_{j}^{i}$ are used [12,13]. The objective for this sub-problem consists in a simultaneous minimization of the number of turbines in operation and status changes for a given time horizon $A$, in order to establish an operation strategy.

The solution of this Unit Commitment can be obtained by solving a mixed integer linear programming optimization problem (MILP) [12] as formulated bellow:

$\operatorname{Min} \sum_{i=1}^{\mathrm{np}} \sum_{j=1}^{\mathrm{ng}}\left(b_{j}^{i} X_{j}^{i}+c_{j}^{i} Y_{j}^{i}+d_{j}^{i} Z_{j}^{i}+\psi P_{\mathrm{ND}_{u}}^{i}+\psi P_{\mathrm{ND}_{L}}^{i}\right)$

s.t.

$-\sum_{j=1}^{\mathrm{ng}} X_{j}^{i} P_{g_{j}}^{\mathrm{Max}} S^{i}+\sum_{k=1}^{\mathrm{nb}} P_{d_{k}}^{i}+P_{\mathrm{LOSS}}^{i}-P_{\mathrm{ND}_{u}}^{i} \leq 0$ for $i=1, \ldots, \mathrm{np}$

$\sum_{j=1}^{\mathrm{ng}} X_{j}^{i} P_{g_{j}}^{\min S^{i}}-\sum_{k=1}^{\mathrm{nb}} P_{d_{k}}^{i}-P_{\text {LOSS }}^{i}-P_{\mathrm{ND}_{L}}^{i} \leq 0$ for $i=1, \ldots, \mathrm{np}$

$\left(X_{j}^{i}-X_{j}^{i-1}\right)-Y_{j}^{i} \leq 0$ for $i=2, \ldots, \mathrm{np}$

$-Y_{j}^{i} \leq 0$ for $i=2, \ldots, \mathrm{np}$

$\left(X_{j}^{i+1}-X_{j}^{i}\right)-Z_{j}^{i} \leq 0$ for $i=1, \ldots, \mathrm{np}-1$

$-Z_{j}^{i} \leq 0$ for $i=1, \ldots, \mathrm{np}-1$

$-P_{\mathrm{ND}_{u}}^{i} \leq 0$ for $i=1, \ldots, \mathrm{np}$

$-P_{\mathrm{ND}_{L}}^{i} \leq 0$ for $i=1, \ldots, \mathrm{np}$ 
The objective function, presented in Eq. (1), corresponds to the minimization of the sum of the product between operational costs $\left(b_{j}^{i}, c_{j}^{i}\right.$ and $\left.d_{j}^{i}\right)$ and the turbine (on/off) status $\left(X_{j}^{i}\right)$, start-up and shutdown $\left(Y_{j}^{i}\right.$ and $Z_{j}^{i}$ ) of each turbine. In this objective function it is considered the possibility that the unit commitment problem in wind parks can present unfeasibility problems because it is not assured that the total available wind power will be greater than the active power requested plus internal wind park losses, for all time intervals, which is different from the conventional cases where the active power generation available is always greater than active power demanded plus any power station internal active losses. To avoid unfeasible solutions for the whole problem, two errors indicators $P_{\mathrm{ND}_{u}}^{i}$ and $P_{\mathrm{ND}_{L}}^{i}$, are then included into the objective function and in the feasibility constrains (2) and (3) of the unit commitment problem. $P_{\mathrm{ND}_{u}}^{i}$ and $P_{\mathrm{ND}_{L}}^{i}$ are affected by a coefficient $\psi$, that is greater than the sum of the other components of the objective function, to assure that $P_{\mathrm{ND} u}^{i}$ and $P_{\mathrm{ND}_{L}}^{i}$ will be zero when the active power requested to the wind park can be generated from the available wind turbines.

Since $P_{\mathrm{ND} u}^{i}$ and $P_{\mathrm{ND}_{L}}^{i}$ are non-negatives variables, as imposed in constraints ( 8 ) and (9), they tend to zero in order to minimize the objective function value if the unit commitment problem is feasible, which happens if, for period $i$, the maximum wind park capability is larger than the requested power plus internal losses or the minimum wind park generation capability plus internal losses is less than the requested power, as imposed in Eqs. (2) and (3).

If these conditions do not occur for a given period $i$, these error variables became:

(a) $P_{\mathrm{ND}_{u}}^{i}=-\sum_{j=1}^{\mathrm{ng}} P_{g_{j}}^{\mathrm{Max} S^{i}}+P_{\mathrm{LOSS}}^{i}+\sum_{k=1}^{\mathrm{nb}} P_{d_{k}}^{i}$ and $P_{\mathrm{ND}_{L}}^{i}=0$ if the requested power is larger than the maximum available generation output; or

(b) $P_{\mathrm{ND}_{u}}^{i}=0$ and $P_{\mathrm{ND}_{L}}^{i}=P_{g}^{\min \mathrm{TM}^{i}}-P_{\mathrm{LOSS}}^{i}-\sum_{k=1}^{\mathrm{nb}} P_{d_{k}}^{i}$ if the minimum wind park capability is larger than the requested power plus internal wind park losses.

The lower wind park capability corresponds to the minimum technical limit of one of the generators, $P_{g}^{\min } \mathrm{TM}^{i}$.

In the case (b) there are not an output from wind park, but in case (a) all the wind turbines are committed and $\sum_{k=1}^{\mathrm{nb}} P_{d_{k}}^{i}-P_{\mathrm{ND}_{u}}^{i}-P_{\text {LOSS }}^{i}$ is the maximum active power that can be delivered from the wind park.

Constrains (2) and (3) establish that the solution is feasible, because from these constrains and considering $P_{\mathrm{ND}_{u}}^{i}=0$ and $P_{\mathrm{ND}_{L}}^{i}=$ 0, $\sum_{k=1}^{n b} P_{d_{k}}^{i}+P_{\text {LOSS }}^{i} \leq \sum_{j=1}^{n g} X_{j}^{i} P_{g_{j}}^{\mathrm{Max} S^{i}}$ and $\sum_{j=1}^{\mathrm{ng}} X_{j}^{i} P_{g_{j}}^{\min S^{i}} \leq \sum_{k=1}^{\mathrm{nb}} P_{d_{k}}^{i}$ $+P_{\text {LOSS }}^{i}$

Eqs. (4)-(7) represent the start-up and shut-down constrains for each wind turbine, as defined in Ref. [12].

\subsection{Dispatch of wind turbines}

\subsubsection{DFIG $P-Q$ characteristics}

Generally, the reference value of the active power that a DFIG should generate is established through optimum generation curves, which provide the active power as a function of the generator rotational speed. Such curves are derived as a result of thorough analysis of the wind turbine aerodynamics, and define the maximum mechanical power the DFIG can extract from the wind at any angular speed $[9,14,15]$.

On the other hand, when establishing the reactive power to be generated or absorbed by a DFIG, the major constraint that should be accounted for consists in the excessively high currents that may

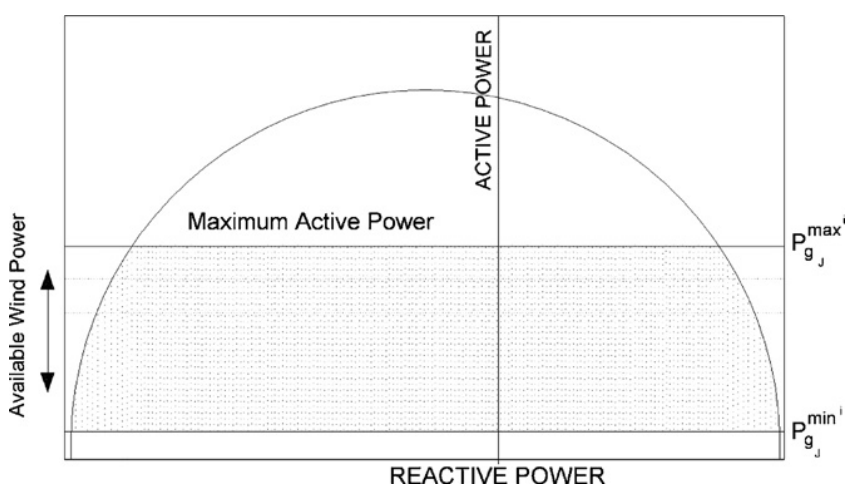

Fig. 2. Capability curves for a DFIG turbine.

be driven into or out of the rotor at any particular ambient temperature [14,15]. From Ref. [14], the following analytical expression referred to as $P-Q$ load curve - relating the stator-side active and reactive powers to the peak value of the rotor current of the $j$ th generator in the $i$ th period, $\left|i_{r_{j}}^{i}\right|$, can be derived:

$\left(P_{\mathrm{g}_{j}}^{i}\right)^{2}+\left(Q_{\mathrm{g}_{j}}^{i}-\frac{3 L_{\mathrm{m}_{j}}\left|V_{j}^{i}\right|^{2}}{4 \pi f_{\mathrm{s}} L_{\mathrm{s}_{j}}}\right)^{2}=\left(\frac{3 L_{\mathrm{m}_{j}}\left|V_{j}^{i}\right|}{2 L_{\mathrm{s}_{j}}}\right)^{2}\left|i_{\mathrm{r}_{j}}^{i}\right|^{2}$

where all the parameters have the same meaning that in Ref. [14]. The examination of (10), reveals that the $P-Q$ load curves correspond approximately to eccentric circumferences in the $P-Q$ plane, whose eccentricity along the $Q$-axis and radius turn out to be $3\left|V_{j}^{i}\right|^{2} / 4 \pi f_{s_{s}} L_{s_{i}}$ and $\left(3 L_{\mathrm{m}_{j}} \mid V_{j}^{i} \| i_{\mathrm{r}_{j}}^{i}\right) / 2 L_{s_{j}}$, respectively [14]. The eccentricity of the curves reveals that the DFIG exhibits a bigger ability to absorb reactive power than to generate it, as expected for any inductive load.

The $P-Q$ characteristic curve for a specified temperature is completed by adding the maximum and minimum active available powers, as defined before, represented as straight lines. Fig. 2 shows the composed curve for the DFIG turbine $P$ - $Q$ output. In this figure the shadow part is the area where the operation of the turbine can be considered as feasible. From this figure, one can observe that when the available active power at the wind turbine is close to its nominal values, the available reactive power decreases. On the other hand, when the available active power at the wind turbine is close to its minimum technical limit, the largest amount of reactive power is available.

\subsubsection{Dispatch problem}

The determination of the active and reactive power generation outputs of each wind turbine for the scheduled turbines that result from the solution of the unit commitment problem, is obtained through the solution of a NLP optimization problem defined through the minimization of the mismatch between the total wind park generation output (active and reactive) and wind park Dispatch Center requests, considering the generator terminal voltage and other operational constrains.

The non-linear optimization sub-problem for each ith interval is formulated as

$\operatorname{Min} \sigma_{P}^{i} \alpha_{P}^{i}+\sigma_{Q}^{i} \alpha_{Q}^{i}$

s.t.

$\left(1-\alpha_{P}^{i}\right) P_{\mathrm{d}_{k}}^{i}-P_{\mathrm{g}_{k}}^{i}+P_{k}^{i}\left(V_{k}^{i}, \theta_{k}^{i}\right)=0$ for $k=1,2, \ldots, \mathrm{nb}$

$\left(1-\alpha_{Q}^{i}\right) Q_{d_{k}}^{i}-Q_{g_{k}}^{i}+Q_{k}^{i}\left(V_{k}^{i}, \theta_{k}^{i}\right)=0$ for $k=1,2, \ldots, n b$

$0 \leq \alpha_{P}^{i} \leq 1$ 
$0 \leq \alpha_{Q}^{i} \leq 1$

$P_{\mathrm{g}_{j}}^{\mathrm{MinS} S^{i}} \leq P_{\mathrm{g}_{j}}^{i} \leq P_{\mathrm{g}_{j}}^{\mathrm{Max} S^{i}} \quad$ for $j=1,2, \ldots, \mathrm{ng}$

$$
Q_{\mathrm{g}_{j}}^{i} \leq\left|\sqrt{\left(\frac{3 L_{\mathrm{m}_{j}}\left|V_{j}^{i}\right|}{2 L_{\mathrm{s}_{j}}}\left|i_{\mathrm{r}_{j}}^{i}\right|\right)^{2}-\left(P_{\mathrm{g}_{j}}^{i}\right)^{2}}\right|
$$

$$
-\frac{3\left|V_{j}^{i}\right|^{2}}{4 \pi f_{s} L_{s_{j}}} \quad \text { for } j=1,2, \ldots, \text { ng }
$$

$$
\begin{gathered}
-Q_{\mathrm{g}_{j}}^{i} \leq\left|\sqrt{\left(\frac{3 L_{\mathrm{m}_{j}}\left|V_{j}^{i}\right|}{2 L_{\mathrm{s}_{j}}}\left|i_{\mathrm{r}_{j}}^{i}\right|\right)^{2}-\left(P_{\mathrm{g}_{j}}^{i}\right)^{2}}\right| \\
+\frac{3\left|V_{j}^{i}\right|^{2}}{4 \pi f_{\mathrm{s}} L_{\mathrm{s}_{j}}} \text { for } j=1,2, \ldots, \mathrm{ng}
\end{gathered}
$$

$V_{k}^{\min } \leq V_{k}^{i} \leq V_{k}^{\operatorname{Max}}$ for $k=1,2, \ldots, \mathrm{nb}$

Eqs. (12) and (13) represent the active and reactive power balance equations in each wind park bus, parameterized by $\alpha_{P}$ and $\alpha_{Q}$ as in $[16,17]$. Since $P_{\mathrm{d}_{k}}^{i}$ and $Q_{\mathrm{d}_{k}}^{i}$ are the wind park output, they are different from zero only when $k$ is the interconnection bus. $P_{g_{k}}^{i}$ and $Q_{g_{k}}^{i}$ are the active and reactive power generated at each wind turbine, and they are different from zero only if the bus is a generation bus and the turbine is committed, and $P_{k}^{i}\left(V_{k}^{i}, \theta_{k}^{i}\right)$ and $Q_{k}^{i}\left(V_{k}^{i}, \theta_{k}^{i}\right)$ are the active and reactive power balance vectors as function of voltages and angles for all the wind park buses.

Eq. (11) shows the objective function adopted, that consists in two variables $\left(\alpha_{P}^{i}\right.$ and $\alpha_{Q}^{i}$ ) representing the percentage of the non-delivered active and reactive power outputs $\left(\alpha_{P}^{i} P_{\mathrm{d}_{k}}^{i}\right.$ and $\left.\alpha_{Q}^{i} Q_{\mathrm{d}_{k}}^{i}\right)$ regarding the Dispatch Center request at each $i$ period. Other terms can be added to the objective function modelling different strategies, as described in [9]. Coefficients $\sigma_{P}^{i}$ and $\sigma_{Q}^{i}$ are weight factors that control the priorities regarding active and reactive power generation.

Limits are imposed in Eqs. (14) and (15) to variables $\alpha$. When $\alpha_{P}^{i}=0$ or $\alpha_{Q}^{i}=0$ it corresponds to the maximum power delivered to the system, as required by the wind park Dispatch Center. On the other side when these variables are equal to 1 , no energy is delivered to the system [16].

Operational limits are considered in Eqs. (16)-(19).

Generated active and reactive powers are accounted in Eqs. (16)-(18), considering the wind turbines operational limits, as available active power, as result of the approach described in Ref. [10], and the minimum technical limits of generators.

Reactive power is treated as a functional variable, such that $Q_{\mathrm{g}_{k}}^{i}=\left(1-\alpha_{Q}^{i}\right) Q_{\mathrm{d}_{k}}^{i}+Q_{k}^{i}\left(V_{k}^{i}, \theta_{k}^{i}\right)$ and maximum and minimum reactive power limits are defined, exploiting Eq. (10), in Eqs. (17) and (18), respectively. For a pre-specified temperature, maximum rotor current $\left|i_{\mathrm{r}_{j}}^{i}\right|$ allowed by the wind turbine is defined, being the limit for reactive power dependent of the stator voltage and the active power generated. As different types of DFIG generators or different temperatures can be considered, different values for $\left|i_{\mathbf{r}_{j}}^{i}\right|$ need to be taken into account. Note that Eqs. (16)-(18) represent the operational area showed in Fig. 2.

Finally, voltage limits for all the buses are considered in Eq. (19).

Line capacity restrictions were not considered since it was assumed that the wind park electrical cables were selected

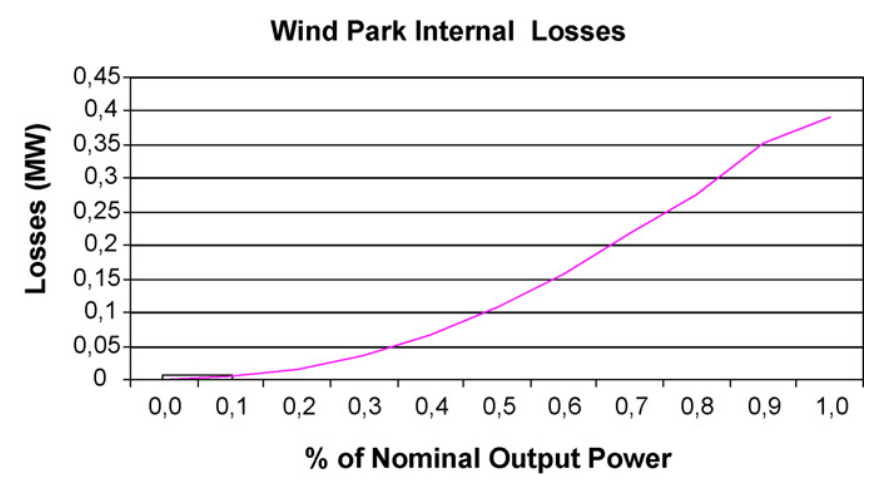

Fig. 3. Losses of wind park for different load request.

accounting properly for the maximum line flows that result from maximum wind power generation.

The NLP sub-problem is solved in this work using the predictorcorrector version of primal-dual non-linear interior points method, as described in Ref. [18].

\section{Results}

Results are present next to illustrate the capabilities of this approach, using a small wind park with 10 turbines (660 kW nominal capacity) and 21 lines forming two feeders connected to the grid interconnection bus through a transformer, as shown in Fig. 1.

For this case it was assumed that each wind generator would be disconnected with its own transformer, without the need of any internal wind park grid reconfiguration.

Voltage limits ( $V_{k}^{\min }$ and $V_{k}^{\mathrm{Max}}$ ) are assumed to be within the range 0.9-1.1 p.u. Limits for active power and reactive power are calculated as described before. The values presented in reference [14] were used to define the $P-Q$ curves of the wind generators used in this research. No capacitor devices are considered. Different available wind active powers are considered for each wind turbine assuming that wind power forecasts are available.

Matlab 7.0 functionalities were used to provide the numerical solutions of the full optimization problem.

Since estimates of the wind park internal losses are needed to solve the unit commitment problem, such an estimate was calculated by interpolating the value of the Dispatch Center request into a curve of losses obtained previously. Fig. 3 shows this curve assuming that the typical relation between reactive power and active power $(\operatorname{tg}(\delta))$ is 0.2 for each wind generator.

\subsection{A general case}

Table 1 shows four maximum available active power values for each wind turbine, obtained from a wind power forecast for four consecutive periods of time. The first column shows the number of the time period, and the others columns show the power available at each generator in each period. These maximum available active power limits result from a wind power forecasting, as described in

Table 1

Maximum power available in each turbine (MW)

\begin{tabular}{llllllllllll}
\hline Period & \multicolumn{2}{l}{ Turbine } & \multicolumn{1}{l}{ Tullal } \\
\cline { 2 - 10 } & 4 & 6 & 8 & 10 & 12 & 14 & 16 & 18 & 20 & 22 & \\
\hline 1 & 0.60 & 0.50 & 0.52 & 0.50 & 0.66 & 0.66 & 0.63 & 0.54 & 0.59 & 0.66 & 5.86 \\
2 & 0.40 & 0.42 & 0.39 & 0.38 & 0.28 & 0.39 & 0.25 & 0.37 & 0.29 & 0.44 & 3.60 \\
3 & 0.37 & 0.39 & 0.45 & 0.57 & 0.52 & 0.56 & 0.58 & 0.44 & 0.37 & 0.51 & 4.75 \\
4 & 0.60 & 0.52 & 0.46 & 0.64 & 0.66 & 0.56 & 0.56 & 0.66 & 0.60 & 0.51 & 5.77 \\
\hline
\end{tabular}


Table 2

Turbine dispatch $\left(P_{\mathrm{g}_{k}}^{i}\right.$ and $\left.Q_{\mathrm{g}_{k}}^{i}\right)$

\begin{tabular}{|c|c|c|c|c|c|c|c|c|}
\hline \multirow[t]{3}{*}{ Turbine bus } & \multicolumn{8}{|l|}{ Intervals } \\
\hline & \multicolumn{2}{|l|}{1} & \multicolumn{2}{|l|}{2} & \multicolumn{2}{|l|}{3} & \multicolumn{2}{|l|}{4} \\
\hline & $P_{\mathrm{g}_{k}}^{1}(\mathrm{MW})$ & $Q_{g_{k}}^{1}($ Mvar $)$ & $P_{\mathrm{g}_{k}}^{2}(\mathrm{MW})$ & $Q_{g_{k}}^{2}$ (Mvar) & $P_{g_{k}}^{3}(\mathrm{MW})$ & $Q_{g_{k}}^{3}($ Mvar $)$ & $P_{\mathrm{g}_{k}}^{4}(\mathrm{MW})$ & $Q_{g_{k}}^{4}($ Mvar $)$ \\
\hline 4 & 0.60 & 0.53 & 0.40 & 0.62 & 0.37 & 0.60 & 0.60 & 0.53 \\
\hline 6 & 0.50 & 0.53 & 0.42 & 0.62 & 0.39 & 0.59 & 0.52 & 0.53 \\
\hline 8 & 0.52 & 0.20 & 0.39 & 0.01 & 0.45 & 0.09 & 0.46 & 0.20 \\
\hline 10 & 0.50 & 0.20 & 0.38 & 0.01 & 0.57 & 0.10 & 0.56 & 0.20 \\
\hline 12 & 0.54 & 0.02 & 0.28 & 0.00 & 0.52 & 0.02 & 0.54 & 0.02 \\
\hline 14 & 0.54 & 0.02 & 0.39 & 0.01 & 0.56 & 0.02 & 0.53 & 0.02 \\
\hline 16 & 0.52 & 0.02 & 0.25 & 0.00 & 0.58 & 0.02 & 0.52 & 0.02 \\
\hline 18 & 0.52 & 0.02 & 0.37 & 0.01 & 0.44 & 0.01 & 0.51 & 0.02 \\
\hline 20 & 0.51 & 0.01 & 0.29 & 0.00 & 0.37 & 0.01 & 0.51 & 0.01 \\
\hline 22 & 0.51 & 0.01 & 0.44 & 0.01 & 0.51 & 0.01 & 0.50 & 0.01 \\
\hline Total & 5.25 & 1.55 & 3.60 & 1.30 & 4.75 & 1.47 & 5.25 & 1.55 \\
\hline Output & 5.00 & 1.00 & 3.48 & 1.00 & 4.54 & 1.00 & 5.00 & 1.00 \\
\hline
\end{tabular}

Ref. [10]. The minimum active power limits correspond to the minimum technical limits of the wind turbines, which were considered as $0.072 \mathrm{MW}$.

A constant Dispatch Center request of $5.0 \mathrm{MW} / 1.0$ Mvar for all these periods was assumed for these tests.

Results for the unit commitment stage show that all the turbines in the wind park of Fig. 1 are committed, and for periods 2 and 3 there are, respectively, 1.65 and $0.50 \mathrm{MW}$ that exceeds the available power at the wind park, considering an amount of estimate losses of $0.248 \mathrm{MW}$, identified as described in Fig. 3. The numerical value used for $\psi$ was 10000 for this case, and values for $b_{j}^{i}, c_{j}^{i}$ and $d_{j}^{i}$ were respectively equal to $1,100,100$ for all $i$ and $j$.

Table 2 shows the turbine dispatch for active and reactive powers and Table 3 the module and angle of voltage in all buses. Turbine numbers are in the first column and the active and reactive powers for each time period are presented in the other columns. In the last row the wind park generation output is shown. Observe that the active power losses estimative are close to the final result. The reactive power output presents no reductions regarding the original Dispatch Center request, showing the availability of reactive power generation when active power is not close to nominal value, as shown in Fig. 2. The non-linear dispatch algorithm tries to reduce the active power losses by reducing the reactive power circulation into wind park, and the buses that are close to the interconnection bus are the ones that generate the larger values of reactive power, complying with the generating limits. This behaviour can be explained considering that the start-up and shut-down costs are fixed in the Unit Commitment stage and in this case the operating costs of the wind park are supposed equal for all the generators, so the effect of reduce the output of each generator maintaining the wind park output is related to reduce the total amount of losses in the park. The $P-Q$ curve explains the capacity to generate high quantities of reactive power when the available active power is far from its maximum. In the same way, the voltages of turbine buses close to the interconnection bus present larger values, in order to allow for an increase of the maximum limit of available reactive power. Voltage at the interconnection bus is fixed at 1 p.u., and the voltage differences between this bus and buses 4 and 6 result from the presence of the transformer installed between buses 1 and 2 . The coefficients adopted for the solution of the non-linear problem considered for this case were $\sigma_{Q}^{i}=1000$ and $\sigma_{Q}^{i}=100$ for all period $i$.

\subsection{Strategies to commit wind turbines}

As explained before, by using different values for parameters $b_{j}^{i}, c_{j}^{i}$ and $d_{j}^{i}$ it is possible to obtain different strategies to commit the turbines. If $b_{j}^{i} \ll c_{j}^{i}$ and $b_{j}^{i} \ll d_{j}^{i}$, the result of the unit commitment problem leads to a minimum changes of status strategy, but if $b_{j}^{i} \gg c_{j}^{i}$ and $b_{j}^{i} \gg d_{j}^{i}$ the result tends to favour a minimum number of turbines in operation at each interval. The following example compares these two strategies.

Tables 5 and 6 present the turbines committed for the different strategies, considering the maximum available power of Table 1 , the technical minimum considered previously and the Dispatch Center request of Table 4 .

As it can be seen from Tables 5 and 6, when the Dispatch Center request is less than the available active power at the wind park, the number of possible combinations implies in different solutions for the unit commitment problem. In the present case, the two strate-

Table 3

Turbine dispatch (voltage and angle)

\begin{tabular}{|c|c|c|c|c|c|c|c|c|}
\hline \multirow[t]{3}{*}{ Turbine bus } & \multicolumn{8}{|l|}{ Intervals } \\
\hline & \multicolumn{2}{|l|}{1} & \multicolumn{2}{|l|}{2} & \multicolumn{2}{|l|}{3} & \multicolumn{2}{|l|}{4} \\
\hline & $V_{k}^{1}$ (p.u.) & $\theta_{k}^{1}\left({ }^{\circ}\right)$ & $V_{k}^{2}$ (p.u.) & $\theta_{k}^{2}\left({ }^{\circ}\right)$ & $V_{k}^{3}$ (p.u.) & $\theta_{k}^{3}\left({ }^{\circ}\right)$ & $V_{k}^{4}$ (p.u.) & $\theta_{k}^{4}\left({ }^{\circ}\right)$ \\
\hline 4 & 1.10 & 0.00 & 1.09 & 0.00 & 1.10 & 0.00 & 1.10 & 0.00 \\
\hline 6 & 1.10 & -0.32 & 1.09 & 0.06 & 1.10 & 0.08 & 1.10 & -0.25 \\
\hline 8 & 1.08 & -0.23 & 1.06 & 0.01 & 1.07 & 0.31 & 1.08 & -0.43 \\
\hline 10 & 1.08 & -0.31 & 1.06 & 0.01 & 1.07 & 0.73 & 1.08 & -0.09 \\
\hline 12 & 1.08 & -0.16 & 1.06 & -0.34 & 1.07 & 0.55 & 1.08 & -0.16 \\
\hline 14 & 1.08 & -0.16 & 1.06 & 0.04 & 1.07 & 0.67 & 1.08 & -0.17 \\
\hline 16 & 1.08 & -0.22 & 1.06 & -0.46 & 1.07 & 0.75 & 1.08 & -0.23 \\
\hline 18 & 1.08 & -0.22 & 1.06 & -0.03 & 1.07 & 0.28 & 1.08 & -0.23 \\
\hline 20 & 1.08 & -0.26 & 1.06 & -0.31 & 1.07 & 0.06 & 1.08 & -0.26 \\
\hline 22 & 1.08 & -0.25 & 1.06 & 0.19 & 1.07 & 0.52 & 1.08 & -0.27 \\
\hline
\end{tabular}


Table 4

Dispatch Center request

\begin{tabular}{ll}
\hline Intervals & \\
1 & 2.0 \\
$P_{\mathrm{d}_{1}}^{1}(\mathrm{MW})$ & 2.0 \\
$Q_{\mathrm{d}_{1}}^{1}($ Mvar $)$ & \\
$2 \quad$ & 3.0 \\
$P_{\mathrm{d}_{1}}^{2}(\mathrm{MW})$ & 3.0 \\
$Q_{\mathrm{d}_{1}}^{2}($ Mvar $)$ & \\
$3 \quad$ & 1.5 \\
$P_{\mathrm{d}_{1}}^{3}(\mathrm{MW})$ & 1.5 \\
$Q_{\mathrm{d}_{1}}^{3}($ Mvar $)$ & \\
$4 \quad$ & 2.0 \\
$P_{\mathrm{d}_{1}}^{4}($ MW) & 2.0 \\
$Q_{\mathrm{d}_{1}}^{4}($ Mvar $)$ & \\
\hline
\end{tabular}

Table 5

Turbines committed (minimum quantity of turbines)

\begin{tabular}{lllll}
\hline Turbine & \multicolumn{2}{l}{ Intervals } & 3 & 4 \\
\cline { 2 - 5 } & 1 & 2 & 0 & 0 \\
\hline 4 & 0 & 1 & 1 & 0 \\
6 & 0 & 1 & 0 & 1 \\
8 & 0 & 1 & 1 & 1 \\
10 & 0 & 1 & 0 & 1 \\
12 & 1 & 1 & 0 & 0 \\
14 & 1 & 1 & 1 & 0 \\
16 & 0 & 0 & 0 & 1 \\
18 & 0 & 1 & 0 & 0 \\
20 & 1 & 1 & 0 & 0 \\
22 & 1 & 1 & 3 & 4 \\
No. of turbines & 4 & 9 & &
\end{tabular}

Table 6

Turbines committed (minimum quantity of changes)

\begin{tabular}{lllll}
\hline Turbine & \multicolumn{2}{l}{ Intervals } & & \\
\cline { 2 - 5 } & 1 & 2 & 1 & 4 \\
\hline 4 & 1 & 1 & 1 & 1 \\
6 & 1 & 1 & 1 & 1 \\
8 & 1 & 1 & 1 & 1 \\
10 & 1 & 1 & 1 & 1 \\
12 & 1 & 1 & 1 & 1 \\
14 & 1 & 1 & 1 & 1 \\
16 & 1 & 1 & 1 & 1 \\
18 & 1 & 1 & 0 & 1 \\
20 & 0 & 0 & 1 & 0 \\
22 & 1 & 1 & 9 & 1 \\
No. of turbines & 9 & 9 & & 9 \\
\hline
\end{tabular}

gies differ in a considerable number of turbines committed, and the relation between cost for start-up/shut-down and turbine operating cost determine the number of turbines in operation. Table 5 presents a solution obtained using $b_{j}^{i}=100$ and $c_{j}^{i}$ and $d_{j}^{i}$ equal to 1 , while for Table 6 these values are $b_{j}^{i}=1$ and $c_{j}^{i}$ and $d_{j}^{i}$ equal to 100 for all $i$ and $j$. A numerical value of $\psi$ of 10000 was adopted for the two cases for all time intervals.

Tables 7 and 8 show the dispatch of the wind turbines for the commitments defined in Tables 5 and 6, respectively. The request of reactive power was set very high in this case, in order to show the effect of the reactive power limits incorporated into the nonlinear problem. As mentioned, when the available active power requested is reduced, the amount of available reactive power is high. This can be observed in Table 7 for the turbine 6 at period 3. In this period, active power dispatch is $0.39 \mathrm{MW}$ (maximum available active power), and the reactive power dispatched is $0.73 \mathrm{Mvar}$ (1.87 times the active power available).

A similar result appears in period 3 in Table 8, with 0.19 MW and 0.79 Mvar dispatched. In all the results obtained for this period, active power generation is distributed among all the generators and dispatched close to their technical minimums. However, reactive power is concentrated only in the generators that are close to the interconnection bus to allow for a reduction of the wind park internal active losses.

The coefficients for non-linear problem objective function considered for this case were $\sigma_{P}^{i}=1000$ and $\sigma_{Q}^{i}=100$ for all $i$.

To decide for a one strategy over another it is necessary to consider the amount of active and reactive power reserves associated to each scheduled turbine. In Table 9, the maximum capabilities of wind park for each turbine commitment is shown. In this table, columns 2 and 4 show the full amount of active power with the commitment of turbines shown in Tables 5 and 6, respectively. As it can be seen, the strategy of minimum status changes allows increasing the active and reactive power reserves in periods 1,2 and 4 . This fact needs to be considered along with the active wind power forecasting confidence interval associated to each dispatch in order to cope with the natural intermittency of the total wind park generation output.

\subsection{Influence of $\sigma_{P}^{i}$ and $\sigma_{Q}^{i}$ values}

As it can be seen from Fig. 2, the most restrictive area to deliver reactive power is close the nominal active power. For this reason, set points for a Dispatch Center request of $6.6 \mathrm{MW}$ and 3.3 Mvar were calculated, considering that all the turbines have the nominal active power available, using different values for $\sigma_{P}^{i}$ and $\sigma_{Q}^{i}$.

Table 7

Turbine dispatch $\left(P_{\mathrm{g}_{k}}^{i}\right.$ and $Q_{\mathrm{g}_{k}}^{i}$ ) for minimum quantity of turbines

\begin{tabular}{|c|c|c|c|c|c|c|c|c|}
\hline \multirow[t]{3}{*}{ Turbine } & \multicolumn{8}{|l|}{ Intervals } \\
\hline & \multicolumn{2}{|l|}{1} & \multicolumn{2}{|l|}{2} & \multicolumn{2}{|l|}{3} & \multicolumn{2}{|l|}{4} \\
\hline & $P_{\mathrm{g}_{k}}^{1}(\mathrm{MW})$ & $Q_{g_{k}}^{1}$ (Mvar) & $P_{\mathrm{g}_{k}}^{2}(\mathrm{MW})$ & $Q_{\mathrm{g}_{k}}^{2}(\mathrm{Mvar})$ & $P_{\mathrm{g}_{k}}^{3}(\mathrm{MW})$ & $Q_{g_{k}}^{3}$ (Mvar) & $P_{\mathrm{g}_{k}}^{4}(\mathrm{MW})$ & $Q_{g_{k}}^{4}$ (Mvar) \\
\hline 4 & & & 0.40 & 0.53 & & & & \\
\hline 6 & & & 0.40 & 0.52 & 0.39 & 0.73 & & \\
\hline 8 & & & 0.38 & 0.50 & & & 0.46 & 0.68 \\
\hline 10 & & & 0.37 & 0.47 & 0.57 & 0.50 & 0.55 & 0.60 \\
\hline 12 & 0.53 & 0.62 & 0.28 & 0.48 & & & 0.54 & 0.52 \\
\hline 14 & 0.53 & 0.62 & 0.36 & 0.43 & & & & \\
\hline 16 & & & & & 0.58 & 0.44 & & \\
\hline 18 & & & 0.35 & 0.28 & & & 0.53 & 0.44 \\
\hline 20 & 0.51 & 0.51 & 0.29 & 0.18 & & & & \\
\hline 22 & 0.51 & 0.51 & 0.34 & 0.02 & & & & \\
\hline Total & 2.09 & 2.24 & 3.17 & 3.40 & 1.54 & 1.66 & 2.08 & 2.24 \\
\hline Output & 2.00 & 2.00 & 3.00 & 3.00 & 1.50 & 1.50 & 2.00 & 2.00 \\
\hline
\end{tabular}


Table 8

Turbine dispatch $\left(P_{\mathrm{g}_{k}}^{i}\right.$ and $\left.Q_{\mathrm{g}_{k}}^{i}\right)$ for minimum quantity of changes

\begin{tabular}{|c|c|c|c|c|c|c|c|c|}
\hline \multirow[t]{3}{*}{ Turbine } & \multicolumn{8}{|l|}{ Intervals } \\
\hline & \multicolumn{2}{|l|}{1} & \multicolumn{2}{|l|}{2} & \multicolumn{2}{|l|}{3} & \multicolumn{2}{|l|}{4} \\
\hline & $P_{\mathrm{g}_{k}}^{1}(\mathrm{MW})$ & $Q_{g_{k}}^{1}$ (Mvar) & $P_{\mathrm{g}_{k}}^{2}(\mathrm{MW})$ & $Q_{g_{k}}^{2}$ (Mvar) & $P_{\mathrm{g}_{k}}^{3}(\mathrm{MW})$ & $Q_{g_{k}}^{3}$ (Mvar) & $P_{\mathrm{g}_{k}}^{4}(\mathrm{MW})$ & $Q_{\mathrm{g}_{k}}^{4}$ (Mvar) \\
\hline 4 & 0.25 & 0.36 & 0.40 & 0.51 & 0.19 & 0.76 & 0.25 & 0.36 \\
\hline 6 & 0.22 & 0.36 & 0.41 & 0.50 & 0.19 & 0.76 & 0.23 & 0.36 \\
\hline 8 & 0.22 & 0.28 & 0.39 & 0.48 & 0.18 & 0.05 & 0.21 & 0.28 \\
\hline 10 & 0.21 & 0.27 & 0.38 & 0.45 & 0.17 & 0.05 & 0.25 & 0.27 \\
\hline 12 & 0.25 & 0.23 & 0.28 & 0.46 & 0.17 & 0.00 & 0.25 & 0.23 \\
\hline 14 & 0.24 & 0.20 & 0.37 & 0.41 & 0.16 & 0.00 & 0.22 & 0.20 \\
\hline 16 & 0.24 & 0.20 & 0.25 & 0.26 & 0.17 & 0.00 & 0.23 & 0.20 \\
\hline 18 & 0.21 & 0.15 & 0.35 & 0.32 & 0.16 & 0.00 & 0.24 & 0.16 \\
\hline 22 & 0.24 & 0.13 & 0.35 & 0.01 & 0.16 & 0.00 & 0.21 & 0.13 \\
\hline Total & 2.08 & 2.18 & 3.17 & 3.40 & 1.54 & 1.64 & 2.08 & 2.18 \\
\hline
\end{tabular}

Table 9

Maximum active and reactive power output

\begin{tabular}{llll}
\hline Period & \multicolumn{2}{l}{ Available wind power } & Minimum no. of changes \\
\cline { 2 - 4 } & \multicolumn{2}{l}{ Minimum no. of turbines } & $\frac{\sum_{j=1}^{10} X_{j}^{i} P_{\mathrm{g}_{j}}^{\mathrm{Max} S^{i}}(\mathrm{MW})}{\sum_{j=1}^{10} X_{j}^{i} P_{\mathrm{g}_{j}}^{\mathrm{Max} S^{i}}(\mathrm{MW})}$ \\
\hline 1 & 2.48 & $\sum_{j=1}^{10} X_{j}^{i} Q_{\mathrm{g}_{j}}^{\mathrm{Max} S^{i}}(\mathrm{Mvar})$ & 4.95 \\
2 & 3.13 & 1.55 & 3.09 \\
3 & 1.50 & 3.62 & 4.11 \\
4 & 2.34 & 1.68 & 4.86 \\
\hline
\end{tabular}

Table 10

Wind Park output as function of $\sigma_{P}^{i}$ and $\sigma_{Q}^{i}$ coefficients

\begin{tabular}{|c|c|c|c|c|c|c|}
\hline \multirow[t]{2}{*}{ Bus } & \multicolumn{2}{|c|}{$\sigma_{P}^{i}=1000$ and $\sigma_{Q}^{i}=100$} & \multicolumn{2}{|c|}{$\sigma_{P}^{i}=1000$ and $\sigma_{Q}^{i}=1.0$} & \multicolumn{2}{|c|}{$\sigma_{P}^{i}=1.0$ and $\sigma_{Q}^{i}=1000$} \\
\hline & $P_{\mathrm{g}_{k}}^{1}(\mathrm{MW})$ & $Q_{g_{k}}^{1}$ (Mvar) & $P_{g_{k}}^{1}(\mathrm{MW})$ & $Q_{\mathrm{g}_{k}}^{1}($ Mvar $)$ & $P_{g_{k}}^{1}(\mathrm{MW})$ & $Q_{g_{k}}^{1}$ (Mvar) \\
\hline 4 & 0.66 & 0.42 & 0.66 & 0.394 & 0.66 & 0.42 \\
\hline 6 & 0.66 & 0.42 & 0.66 & 0.394 & 0.66 & 0.42 \\
\hline 8 & 0.66 & 0.34 & 0.66 & 0.027 & 0.66 & 0.41 \\
\hline 10 & 0.66 & 0.34 & 0.66 & 0.027 & 0.66 & 0.41 \\
\hline 12 & 0.66 & 0.27 & 0.66 & 0.026 & 0.66 & 0.39 \\
\hline 14 & 0.66 & 0.27 & 0.66 & 0.026 & 0.66 & 0.39 \\
\hline 16 & 0.66 & 0.23 & 0.66 & 0.025 & 0.17 & 0.37 \\
\hline 18 & 0.66 & 0.23 & 0.66 & 0.025 & 0.17 & 0.37 \\
\hline 20 & 0.66 & 0.21 & 0.66 & 0.025 & 0.01 & 0.37 \\
\hline 22 & 0.66 & 0.21 & 0.66 & 0.025 & 0.01 & 0.37 \\
\hline Total & 6.60 & 2.95 & 6.60 & 0.99 & 4.32 & 3.93 \\
\hline
\end{tabular}

Table 10 shows the solutions for the active and reactive powers generated for $\sigma_{P}^{i}=1000$ and $\sigma_{Q}^{i}=100, \sigma_{P}^{i}=1000$ and $\sigma_{Q}^{i}=1$, $\sigma_{P}^{i}=1$ and $\sigma_{Q}^{i}=1000$ at each turbine. The active power generated at each wind turbine is shown in columns 2,4 and 6 , and the reactive power at columns 3, 5 and 7 .

Since the Wind Park output needs to consider the internal active and reactive power losses, the amount of active and reactive power delivered to the grid varies in each case. For the first case, this is $6.18 \mathrm{MW}$ and $2.05 \mathrm{Mvar}$. In the second case, they are $6.23 \mathrm{MW}$ and $0.19 \mathrm{Mvar}$, and for the last case $4.05 \mathrm{MW}$ and 3.3 Mvar, fulfilling the reactive power request. As it can be seen, in the second case the wind turbines placed far away from bus 1 (interconnection bus) have their reactive power output more reduced than turbines at buses 4 and 6 . On the other hand, when $\sigma_{P}^{i}=1$ and $\sigma_{O}^{i}=$ 1000 , reactive power output has priority over active power and can be observed that turbines 16, 18, 20 and 22 have their active power output reduced in order to guarantee the reactive power output.

The value of active or reactive powers generated in order to reduce internal losses while delivering the maximum active or reac- tive powers depends therefore on the $P-Q$ characteristic of the wind generators and on the type of priority decisions that the wind park managing system adopts.

\section{Conclusion}

In this paper, a global optimization strategy was developed for the identification of the commitment of wind turbines in a wind park, as well as their active and reactive power set points, using a combination of MILP and NLP optimization techniques. This strategy allows following Wind Park Dispatch Center requests, regarding active/reactive power to be generated. The approach was tested with a small wind park having 10 generators and with different wind power availability scenarios and generation requests. Results obtained proved the effectiveness of the developed approach, demonstrating its practical value in cases where wind parks are requested to follow specific time sequence generation profiles. The proposed approach is flexible enough to be used for different operational strategies. In the Unit Commitment problem, parameters $b_{j}^{i}, c_{j}^{i}$ and $d_{j}^{i}$ can assume any values in order to follow a pre-defined 
strategy, avoiding turbines switching off. In the Dispatch problem, the capability curves of the DFIG of the wind park are taken into account, allowing the wind park to be effectively committed to voltage control defined by the TSO or DSO.

The use of a confidence interval regarding active power generation restricts the solution of the optimization problem, requiring a more complex analysis scheme that needs to be investigated, since the relation between available active power and active power to be dispatched is highly non-linear.

\section{Acknowledgements}

C.F. Moyano thanks for the Financial support granted by Fundação para a Ciência e a Tecnologia (FCT) and Instituto de Engenharia de Sistemas e Computadores do Porto (INESC), Portugal, where this work was developed.

\section{Appendix A. List of symbols and notations}

$b_{j}^{i} \quad$ cost associated to maintaining on turbine $j$ at period $i$

$c_{j}^{i} \quad$ cost of start-up for turbine $j$ at period $i$

$d_{j}^{i} \quad$ cost of shut-down for turbine $j$ at period $i$

DSO distribution system operators

DFIG double fed induction generators

$f_{\mathrm{s}} \quad$ grid frequency (considered constant for all periods)

$i \quad$ time period considered

$i_{\mathrm{r}_{j}}^{i} \quad$ peak value of the rotor current for turbine $j$ at period $i$

$j$ turbine number

$k \quad$ bus number

$L_{\mathrm{m}_{j}} \quad$ magnetizing inductance for turbine $j$

$L_{\mathrm{s}_{j}} \quad$ stator inductance for turbine $j$

MILP mixed integer linear programming optimization problem

$\mathrm{nb} \quad$ number of buses in the wind park

ng number of wind generators

np number of time periods

OPF optimal power flow

$P_{\mathrm{d}_{k}}^{i} \quad$ active power required by the Dispatch Center at the interconnection buses for time interval $i$. If $k$ is not a interconnection bus, $P_{\mathrm{d}_{k}}^{i}=0$

$P_{g_{j}}^{i} \quad$ active power generated by the wind generator for turbine $j$ at period $i$

$P_{k}^{i}\left(V_{k}^{i}, \theta_{k}^{i}\right)$ active power flow equation for bus $k$ of the wind park at period $i$

$P_{\mathrm{LOSS}}^{i} \quad$ estimate for the wind park losses for time interval $i$

$P_{\mathrm{ND}_{L}}^{i} \quad$ free non-negative variable that avoids unfeasibility in unit commitment problem if the load is lower than generation capability for period $i$

$P_{\mathrm{ND}_{u}}^{i} \quad$ free non-negative variable that avoids unfeasibility in unit commitment problem if the load is greater than generation capability for period $i$

$P_{\mathrm{g}_{j}}^{\mathrm{MaxS}} S^{i} \quad$ maximum available active power limit of the generator, determined from the wind power forecasting for turbine $j$ in time interval $i$

$P_{\mathrm{g}_{j}}^{\min S^{i}} \quad$ minimum technical limit for the wind turbine $j$ in time interval $i$, if there is enough wind speed to keep the generator in operation

$P_{\mathrm{g}}^{\min T M^{i}}$ minimum technical capability of wind park at period $i$

$Q_{g_{j}}^{i} \quad$ reactive power generated by the wind generator for turbine $j$ at period $i$

$Q_{k}^{i}\left(V_{k}^{i}, \theta_{k}^{i}\right)$ reactive power flow equation for bus $k$ of the wind park at period $i$

\author{
TSO transmission system operator \\ $V_{k}^{i} \quad$ voltage module at bus $k$ of the wind park at period $i$ \\ $V_{k}^{\mathrm{Max}} \quad$ maximum voltage module at wind park bus $k$. Considered \\ as constant in this research \\ $V_{k}^{\min } \quad$ minimum voltage module at wind park bus $k$. Considered \\ as constant in this research \\ $X_{j}^{i} \quad$ binary variables describing the on/off turbine status for \\ period $i$ and turbine $j$ \\ $Y_{j}^{i} \quad$ start-up action for each turbine $j$ at period $i$ \\ $Z_{j}^{i} \quad$ shut-down actions for each turbine $j$ at period $i$
}

\section{Greek letters}

$\alpha_{P}^{i} \quad$ parameter used to identify the percentage of nondelivered active power at interval $i$

$\alpha_{Q}^{i} \quad$ parameter used to identify the percentage of nondelivered reactive power at interval $i$

$\theta_{k}^{i} \quad$ voltage angle at bus $k$ of the wind park at period $i$

$\pi \quad$ constant, number phi

$\sigma_{P}^{i} \quad$ weight factor that control the priorities regarding active power generation at period $i$

$\sigma_{Q}^{i} \quad$ weight factor that control the priorities regarding reactive power generation at period $i$

\section{Appendix B}

A. General parameters: base values for the pu system: base power: 100 Mva; base voltages: $0.69 \mathrm{kV}$ for LV buses, $15 \mathrm{kV}$ for MV buses, and $63 \mathrm{kV}$ for $\mathrm{HV}$.

B. Wind turbine: cut-in speed $=4 \mathrm{~m} / \mathrm{s}$, cut-off speed $=25 \mathrm{~m} / \mathrm{s}$, $L_{\mathrm{m}}=19.37 \mathrm{mH}, L_{\mathrm{S}}=7.468 \mathrm{mH}$, and $\left|i_{r}\right|=0.0187$ p.u.

C. Transformers: wind generator transformer $(0.69: 15 \mathrm{kV})$ : $\operatorname{Sn}(\mathrm{kva})=750, X_{\mathrm{t}}=5 \%$; wind park transformer $(15: 63 \mathrm{kV})$, $\mathrm{Sn}(\mathrm{Mva})=4.5, X_{\mathrm{t}}=5 \%$.

D. Line parameters: line $1-2+$ wind park transformer $=0.7690+$ $j 1.39356$ p.u. For all lines: line impedance $=0.1986+j 0.0156$ p.u., with a distance of $0.310 \mathrm{~km}$. For each turbine transformer, $X_{\mathrm{t}}=6.667$ p.u., $X_{\mathrm{c}}$ line $=0.00079$ p.u.

\section{References}

[1] A. Estanqueiro, R. Castro, J.R. Medeiros Pinto, R. Rodrigues, J. Peças Lopes, How to prepare a power system for $12 \%$ wind energy penetration: the Portuguese case study, in: Proceedings of the Nordic Wind Power Conference, Espoo, Finland, May 22-23, 2006

[2] Royal Decree 436/2004, Establishing the Methodology to Update and Organize the Legal and Economic Procedures for Electricity Generation Belonging to the Special Regime, March 12, 2004 [Online]. Available: http://www.cne.es.

[3] J.L. Rodriguez-Amenedo, et al., Automatic generation control of a wind farm with variable speed wind turbines, IEEE Trans. Energy Convers. 17 (June (2)) (2002) 279-284

[4] J. Wiik, J.O. Gjerde, T. Gjengedal, M. Gustafsson, Steady state power system issues when planning large wind farms, in: Power Engineering Society Winter Meeting, 2002. IEEE, vol. 1, January 27-31, 2002, pp. 199204.

[5] A. Tapia, G. Tapia, J.X. Ostolaza, J.R. Saenz, R. Criado, J.L. Berasategui, Reactive power control of a wind farm made up with doubly fed induction generators (I and II), in: Proceedings of the 2001 IEEE Porto Power Tech Conference, Porto, Portugal, September 10-13, 2001.

[6] R.G. de Almeida, J.A. Peças Lopes, J.A.L. Barreiros, Improving power system dynamic behavior through doubly fed induction machines controlled by static converter using fuzzy control, IEEE Trans. Power Syst. 19 (November (4)) (2004) 1942-1950.

[7] A.D. Hansen, P. Sørensen, F. Iov, F. Blaabjerg, Centralised power control of wind farm with doubly fed induction generators, Renew. Energy 31 (2006) 935951.

[8] O. Anaya-Lara, F.M. Hughes, N. Jenkins, G. Strbac, Contribution of DFIG-based wind farms to power system short-term frequency regulation, IEE Proc.Gener. Transm. Distrib. 153 (March (2)) (2006) 164-170.

[9] R.G. de Almeida, E.D. Castronuovo, J.A. Peças Lopes, Optimum generation control in wind parks when carrying out system operator requests, IEEE Trans. Power Syst. 21 (May (2)) (2006). 
[10] P. Pinson, G. Kariniotakis, On-line assessment of prediction risk for wind power production forecasts, Wind Energy 7 (April/June (2)) (2004) 119-132.

[11] S.M.V. Rueda, K.C. Almeida, Optimal power flow solutions under variable load conditions: reactive power cost modeling. Optimal power flow solutions under variable load conditions: reactive power cost modeling, in: Proceedings of the Power Industry Computer Applications, 2001. PICA 2001. Innovative Computing for Power - Electric Energy Meets the Market. 22nd IEEE Power Engineering Society International Conference on May 20-24, 2001, 2001, pp. 300-305.

[12] M. Carrión, J.M. Arroyo, A Computationally efficient mixed-integer linear formulation for the thermal unit commitment problem, IEEE Trans. Power Syst. 21 (August (3)) (2006) 1371-1378.

[13] J. Medina, V.H. Quintana, A.J. Conejo, A clipping-off interior point technique for medium-term hydro-thermal coordination, IEEE Trans. Power Syst. 14 (February (1)) (1999) 266-273.

[14] G. Tapia, A. Tapia, J.X. Ostolaza, Two alternative modeling approaches for the evaluation of wind farm active and reactive power performances, IEEE Trans. Energy Convers. 21 (December (4)) (2006) 909-920.

[15] A.Tapia, G. Tapia,J.X. Ostolaza, J.R. Saenz, Modeling and control of a wind turbine driven doubly fed induction generator, IEEE Trans. Energy Convers. 18 (June (2)) (2003) 194-204.

[16] C.F. Moyano, R.S. Salgado, Adjusted OPF solution using parameterized model, automation and control magazine, Braz. Autom. Soc. 17 (April, May and June (2)) (2006) (in Portuguese).

[17] S. Granville, J.C.O. Mello, A.C.G. Melo, Application of interior point methods to power flow unsolvability, IEEE Trans. Power Syst. 11 (May (2)) (1996) 1096-1103.
[18] E.D. Castronuovo, J.M. Campagnolo, R. Salgado, New versions of nonlinear interior point methods applied to the optimal power flow, in: Proceedings of the IEEE T\&D Latin America, São Paulo, Brazil, April, 2002.

[19] A. Rodrigues, J.A.P. Lopes, P. Miranda, J. Palma, C. Monteiro, J.N. Souza, R.J. Bessa, C. Rodrigues, J. Matos, EPREV-A wind power forecasting tool for Portugal, in: Proceedings of the EWEC 2007, Milan, Italy, May 7-10, 2007.

Carlos F. Moyano received the B.Sc. degree in Electrical Engineering in 1993 from the National University of La Plata, Argentina, the M.Sc. and Ph.D. degrees from the Federal University of Santa Catarina, Brazil, in 2001 and 2005, respectively. Between 2006-2007 performed postdoctoral research at Instituto de Engenharia de Sistemas e Computadores do Porto (INESC), Porto, Portugal. Actually is Research Fellow in Power Engineering at Queensland University of Technology, Brisbane, Queensland, Australia.

João A. Peças Lopes (M'80-SM'94) received the Electrical Engineering degree (5-year course), the Ph.D. degree in electrical engineering, and the Aggregation degree from the University of Porto, Porto, Portugal, in 1981, 1988, and 1996, respectively. He is an Associate Professor with Aggregation at the Department of Electrical Engineering, Faculty of Engineering, University of Porto. In 1989, he joined the staff of Instituto de Engenharia de Sistemas e Computadores do Porto (INESC) as a Senior Researcher, and he is presently Coordinator of the Power Systems Unit. 